\title{
REFLEXÕES ACERCA DO PANORAMA DE CONSUMO DE ÁlCOOL E/OU OUTRAS DROGAS ENTRE ESTUDANTES UNIVERSITÁRIOS
}

\section{REFLECTIONS ABOUT THE PANORAMA OF CONSUMPTION OF ALCOHOL AND/OR OTHER DRUGS AMONG UNIVERSITY STUDENTS}

\section{REFLEXIONES SOBRE EL PANORAMA DEL CONSUMO DE ALCOHOL Y OTRAS DROGAS ENTRE ESTUDIANTES UNIVERSITARIOS}

\author{
Jamila Souza Gonçalves ${ }^{1}$, Silvana Maria Coelho Leite Fava ${ }^{2}$, Andrea Cristina Alves ${ }^{3}$, Eliza Maria Resende Dázio ${ }^{4}$.
}

\section{RESUMO}

Objetivo: Refletir acerca do panorama de consumo de álcool e/ou outras drogas entre estudantes universitários e suas implicações na vida acadêmica e pessoal desses estudantes. Método: Trata-se de uma reflexão teórica, construída com base na leitura de estudos científicos atuais que atendem à seguinte questão norteadora: Qual o panorama de consumo de álcool e/ou outras drogas entre estudantes universitários? Os artigos analisados levaram à concretização da seguinte categoria: "Panorama de consumo de álcool e/ou outras drogas entre estudantes universitários. Resultados: Verificou-se que o álcool foi a droga de maior consumo entre os estudantes. Embora exista uma preocupação entre os pesquisadores em relação ao uso de álcool e/ou outras drogas entre universitários ficou evidente que o início do consumo dessas substâncias entre universitários não se encontra, necessariamente, vinculado ao ingresso na universidade. Conclusão: Constatou-se o uso cada vez mais excessivo de substâncias psicoativas entre os jovens universitários, sendo preciso que a universidade cumpra seu papel, criando políticas efetivas para o enfrentamento das questões referentes ao uso de álcool e/ou outras drogas, com vistas às ações de prevenção e de tratamento, que contemplem as reais necessidades dos jovens universitários, sem nenhum tipo de discriminação.

Descritores: Bebidas alcoólicas; Drogas ilícitas; Estudantes.

\section{ABSTRACT}

Objective: to reflect about the panorama of consumption of alcohol and/or other drugs among college students and its implications in their academic and personal life. Method: theoretical reflection, based on the reading of current scientific studies that answer the following guiding question: What is the panorama of alcohol and/or other drugs consumption among university students? The articles analyzed led to the implementation of the following category: "Panorama of consumption of alcohol and/or other drugs among college students. Results: it was found that alcohol was the major drug consumed by students. Although there is a concern among researchers concerning the use of alcohol and/or other drugs among university students, it was evident that the beginning of the consumption of these substances by college students is not necessarily linked to the University. Conclusion: there have been increasingly excessive use of psychoactive drugs among university students, and the University fulfill your role by creating effective policies to deal with the issues relating to the use of alcohol and/or other drugs, with actions related to prevention and treatment related to the real needs of the young university students, without any discrimination.

Descriptors: Alcoholic beverages; Street drugs; Students.

\section{RESUMEN}

Objetivo: reflexionar sobre el panorama del consumo de alcohol y otras drogas entre estudiantes universitarios y sus implicaciones en la vida personal y académica de estos estudiantes. Método: Se trata de una reflexión teórica, construida con base en la lectura de estudios científicos actuales que atienden la siguiente cuestión orientadora: ¿Cuál es el panorama de consumo de alcohol y / o otras drogas entre estudiantes universitarios? Los artículos analizados condujeron a la aplicación de la siguiente categoría: "Panorama del consumo de alcohol y otras drogas entre estudiantes universitarios. Resultados: se encontró que el consumo de alcohol era lo mayor entre los estudiantes. Aunque haya una preocupación entre los investigadores sobre el uso de alcohol y otras drogas entre estudiantes de Universidades, es evidente que el inicio del consumo de estas sustancias entre los estudiantes universitarios no está necesariamente vinculado a las Universidades. Conclusión: ha habido el uso cada vez más excesivo de drogas psicoactivas entre estudiantes de las Universidades. Ellas cumplen con su rol social mediante la creación de políticas eficaces para abordar las cuestiones relacionadas con el uso de alcohol y otras drogas, con vistas a acciones de prevención y tratamiento, necesidades reales de los jóvenes universitarios, sin discriminación alguna.

Descriptores: Bebidas alcohólicas; Drogas ilícitas; Estudiantes.

${ }^{1}$ Doutoranda em Enfermagem Psiquiátrica pela Escola de Enfermagem de Ribeirão Preto - EERP-USP. Docente do Instituto Federal de Educação, Ciência e Tecnologia do Sul de Minas - Campus Passos. ${ }^{2}$ Doutora em Ciências. Docente da Escola de Enfermagem da Universidade Federal de Alfenas - Unifal-MG. ${ }^{3}$ Doutoranda em Enfermagem Psiquiátrica pela Escola de Enfermagem de Ribeirão Preto - EERP-USP. Docente do Instituto Federal de Educação, Ciência e Tecnologia do Sul de Minas - Campus Passos. ${ }^{4}$ Doutora em Enfermagem. Docente da Escola de Enfermagem da Universidade Federal de Alfenas - Unifal-MG.

Como citar este artigo:

Gonçalves JS, Fava SMCL, Alves AC, et al. Reflexões acerca do panorama de consumo de álcool e outras drogas entre estudantes universitários. Revista de Enfermagem do Centro Oeste Mineiro. 2019;9: e2594. [Access___]; Available in: DOI: http://dx.doi.org/10.19175/recom.v9i0.2594 


\section{INTRODUÇÃO}

O uso abusivo de drogas, como bebidas alcoólicas, tabaco e outras substâncias psicoativas (SPA) é um fenômeno mundial considerado problema de saúde pública, com repercussões físicas, mentais, socioeconômico-culturais e legais ${ }^{(1-3)}$.

Dentre essas repercussões do uso abusivo e dependência, estão o sofrimento para a pessoa que consome a droga e para a sua família, prejuízos na vida escolar, profissional, crescimento de delitos e enriquecimento ilícito, além de contribuir com o aumento da carga global de doenças, resultando em perdas por mortes prematuras e anos vividos com incapacidade ${ }^{(3-4)}$.

Estimativas revelam que cerca de 243 milhões pessoas, o equivalente a $5 \%$ da população mundial, com idade entre 15-64 anos, faz uso de drogas ilícitas, pelo menos uma vez no ano. Em termos de substância utilizada, em geral, os homens são três vezes mais propensos ao uso de drogas ilícitas se comparado às mulheres. Em relação ao consumo problemático, o número permanece estável, cerca 27 milhões de pessoas $^{(3)}$.

Nesse contexto, os estudantes universitários têm merecido, por parte de pesquisadores e de profissionais de saúde e de educação, uma atenção especial, visto que no ambiente universitário, o uso de álcool e outras drogas encontra-se cada vez mais difundido. Fatores como saída do núcleo familiar, processo de adaptação ao novo meio, dificuldade de inserção em novos grupos e disponibilidade dessas substâncias, aumentam a vulnerabilidade dos estudantes ao consumo dessas drogas ${ }^{(5-6)}$.

Em termos de gênero, observa-se uma crescente similaridade de consumo de álcool e outras drogas entre homens e mulheres, em especial no ambiente universitário ${ }^{(7-8)}$.

No Primeiro Levantamento Nacional sobre o Uso de Álcool, Tabaco e Outras Drogas entre Universitários, realizado nas 27 capitais brasileiras, $86,2 \%$ dos estudantes relataram ter consumido bebidas alcoólicas e, em relação a outras drogas, $48,7 \%$ disseram já ter feito uso na vida. O mesmo levantamento constatou que $77,3 \%$ dos homens e $66,6 \%$ das mulheres relataram ter feito consumo de bebidas alcoólicas nos últimos 12 meses $^{(9)}$.

A diferença do consumo de substâncias, em especial o álcool, entre homens e mulheres tem se tornado um importante tópico de discussões e pesquisas, tendo em vista as diferentes necessidades, razões e motivações que os levam a consumir bebidas alcoólicas ${ }^{(10)}$. Sendo assim, questiona-se: qual o panorama de consumo de álcool e outras drogas entre estudantes universitários?

Frente ao exposto, busca-se refletir acerca do panorama de consumo de álcool e/ou outras drogas entre estudantes universitários.

\section{MÉTODO}

Trata-se de um estudo de abordagem teórico-reflexiva sobre o consumo de álcool e/ou outras drogas entre universitários e suas implicações na vida acadêmica e pessoal. Para a reflexão foram obtidas publicações de artigos escritos na língua portuguesa, espanhola e inglesa, produzidos nos últimos 15 anos, e que focavam a temática em questão. A justificativa para a escolha desse período ocorreu, em razão da realização de vários levantamentos pelo Centro Brasileiro de Informações sobre Drogas (CEBRID) e pela Secretaria Nacional de Políticas Sobre Drogas (SENAD), a partir de 2001. O levantamento da literatura sobre o referido tema ocorreu nas bases de dados da Literatura LatinoAmericana e do Caribe de Informações em Ciências da Saúde (LILACS), Medical Literature Analysis and Retrieval System Online (MEDLINE), Índice Bibliográfico Espanhol de Ciências da Saúde (IBECS), American Psychological Association (APA) e a National Center for Biotechnology Information (PUBMED) e nas bibliotecas virtuais Scientific Electronic Library Online (SCIELO), Biblioteca Regional de Medicina (BIREME) e Biblioteca Cochrane, com os descritores de Ciências da Saúde (DeCS) e Medical Subject Headings (MESH), "álcool", "drogas", "estudantes" e o operador booleano "and".

Após a leitura e análise dos artigos construiu o tema central: Panorama do consumo de álcool e/ou outras drogas entre universitários.

\section{RESULTADOS E DISCUSSÃO}

\section{Panorama do consumo de álcool e outras} drogas entre universitários

Atualmente, o consumo de SPA tem se tornado um fenômeno frequente, principalmente entre jovens inseridos no contexto universitário.

Estudo quantitativo com o objetivo de averiguar as características cruciais do perfil epidemiológico e de drogadição na população 
universitária da Universidade de El Salvador, por meio da aplicação de um questionário estruturado autoadministrável, constatou-se a prevalência do sexo feminino em relação à população estudada. O álcool foi a droga de maior consumo entre os estudantes, seguido pelo tabaco. Evidenciou-se uma conduta similar entre os sexos quanto ao uso do álcool. Em relação ao tabaco há uma prevalência do sexo feminino. Os autores observaram que a maioria dos estudantes não percebia o impacto do consumo de álcool e/ou outras drogas sob o rendimento acadêmico $^{(11)}$.

Pesquisa desenvolvida com o objetivo de identificar os padrões de Policonsumo Simultâneo de Drogas (PSD) e suas implicações legais, sociais e de gênero entre estudantes universitários de ciências da saúde, constatou numa amostra de 275 estudantes dos cursos de Enfermagem, Medicina, Farmácia, Nutrição, Fisioterapia e Terapia Ocupacional, a ocorrência de PSD em $27,9 \%$ das estudantes. A combinação do álcool e maconha foi observada em $30 \%$ dos estudantes nos últimos dois meses, seguido da combinação de álcool e medicamentos prescritos. O PSD encontrou-se mais acentuado entre homens. Os autores constataram que o PSD entre os estudantes da área da saúde, foram atribuídas às angustias ao lidar com a dor e o sofrimento de seus pacientes, à crise natural da idade e, muitas vezes, ao ingresso precoce na universidade ${ }^{(6)}$.

Estudo quantitativo realizado com 437 estudantes em uma instituição de ensino superior do Vale do Paraíba teve por objetivos identificar o número de universitários que faziam uso de substâncias psicoativas, verificar que fatores influenciavam esse consumo e o número de universitários que necessitavam receber intervenções $^{(12)}$. Evidenciou-se que a primeira experiência com bebidas alcoólicas entre os graduandos ocorreu na faixa etária de 13 a 18 anos, o álcool e o tabaco foram as drogas mais consumidas e a existência de uma relação entre estudantes que faltavam às aulas por estar consumindo álcool no período das aulas. As estudantes, em sua maioria, negaram que tal atitude atrapalhava o desempenho acadêmico ${ }^{(12)}$.

Ao realizar um estudo do tipo quantitativo analítico, prospectivo, transversal e observacional realizado com 345 estudantes, sendo 239 do sexo feminino, cujo objetivo foi determinar o perfil da utilização de drogas lícitas e ilícitas por universitários de uma instituição privada do município de Quixadá, no Ceará, constatou-se que o consumo de drogas como álcool e a cocaína foi prevalente. Os estudantes destacaram a influência de amigos (13\%) e a sensação de diversão (21\%) causada pelas drogas, como principais fatores motivacionais para o seu uso ${ }^{(13)}$. Os autores destacam que $46 \%$ dos estudantes afirmaram que não tinham motivos aparentes para o consumo e $39 \%$ consideraram as festas universitárias propícias ao uso do álcool. Outro fato importante foi que $71 \%$ dos universitários fizeram uso de medicamentos sem prescrição médica. Entre os participantes, o uso de álcool e/ou outras drogas estavam relacionados à renda familiar mais elevada. Para os autores, o ambiente universitário influencia o consumo abusivo de substâncias psicoativas ${ }^{(13)}$.

Ao realizar um estudo do tipo descritivo exploratório, de coorte transversal, com 313 estudantes do primeiro e quarto ano de graduação em Enfermagem cujo objetivo foi analisar a relação entre fatores de risco, níveis de espiritualidade e uso de álcool entre estudantes de dois cursos de enfermagem, constatou-se que os fatores de risco associados ao consumo de álcool foram: sexo masculino, ser casado, ter baixo nível de espiritualidade e não ter filiação religiosa. Os resultados revelaram que o maior consumo de bebidas alcoólicas esteve presente entre o sexo masculino; a espiritualidade não foi considerada um fator de proteção frente ao uso do álcool e a faixa etária dos 20 anos representou a maior parte da população que bebe excessivamente, embriaga-se com regularidade e demonstra problemas com bebida constantemente associados a esse padrão de consumo $^{(14)}$.

Constatou-se ainda que em relação à filiação religiosa, houve diferenças significativas na classificação de AUDIT e a escala de espiritualidade. No entanto, acreditavam que alguns aspectos da religiosidade, como frequentarem igreja, preferência religiosa e o ato de orar, apresentaram influência protetora sobre - consumo de substâncias em estudantes universitários ${ }^{(14)}$. Em contrapartida a esses resultados, outro estudo, constatou que a religião é caracterizada como importante fator de proteção ao consumo de álcool e outras drogas $^{(13)}$.

Estudo de caráter descritivo e delineamento correlacional foi realizado com os objetivos de conhecer os fatores associados ao uso e abuso de álcool entre acadêmicos e relacionar o uso e abuso dessa substância às 
consequências desse hábito. A amostra foi constituída por 351 estudantes do primeiro e penúltimo ano dos cursos da área da saúde, sendo que 238 eram mulheres. Na coleta de dados, os pesquisadores aplicaram um questionário com informações sociodemográficas e o AUDIT. O estudo evidenciou que 202 (57,5\%) estudantes tinham o costume de ingerir bebidas alcoólicas. A idade de início desse consumo ocorreu na faixa etária dos 10 aos 17 anos ${ }^{(15)}$.

Dentre os fatores que mais influenciaram o consumo de bebidas alcoólicas precoce estavam a mídia, o consumo por parte dos familiares e dos amigos. Os autores ressaltaram que o ingresso na universidade, o distanciamento do núcleo familiar e o estabelecimento de novos laços de amizade favorecem a oportunidade para o consumo de álcool e outras drogas, principalmente por parte dos estudantes em maior vulnerabilidade psicossocial. Além disso, o ambiente acadêmico tornou-se propício a experimentação de drogas, uma vez que, nesse momento da vida, o jovem participa de inúmeras atividades e eventos sociais. Dentre as consequências do beber problemático, identificadas nesse estudo, estão os apagões, coma alcoólico e acidentes automobilísticos. Para as autoras, o conhecimento adquirido no decorrer dos anos acadêmicos acerca dos danos ocasionados pelo álcool, no organismo humano, não surtiu efeito sobre consumo entre os estudantes investigados. Sugerem a inserção de discussões nas disciplinas acadêmicas no que se refere à Política Nacional do Álcool acrescida de outras legislações relacionadas, buscando assim, conscientizar os jovens ${ }^{(15)}$.

No estudo quantitativo descritivo que teve por objetivo descrever o uso de drogas entre alunos de primeiro ano universitário, conhecer o padrão de consumo das substâncias usadas e comparar a relação entre o uso de drogas, os comportamentos de risco e o gênero, evidenciouse que a média de idade do primeiro consumo foi de 13 anos. Tais resultados apontaram que 0 consumo de álcool e outras drogas, provavelmente tenha acontecido antes do ingresso na universidade ${ }^{(16)}$.

Resultados similares foram encontrados em outros estudos em que constataram que a primeira experiência com bebidas alcoólicas entre os graduandos ocorreu na faixa etária de 13 a 18 anos. Subentende-se que a universidade não seria, necessariamente, a razão principal pela qual os estudantes iniciaram o consumo de substâncias psicoativas, em especial, o álcool. A utilização dessas substâncias, assim como a frequência, estaria atribuída a um conjunto de fatores que se inter-relacionam desencadeando todo o processo ${ }^{(12-15)}$.

Em relação ao gênero, os homens têm se embriagado mais do que as mulheres, assim como o número de intoxicações por álcool e outras drogas. Quanto ao comportamento do uso dessas substâncias entre estudantes, 53 (26,5\%) dos estudantes gostavam de beber de maneira suficiente para ficar um pouco "alto", enquanto que as estudantes, $37(18,5 \%)$ não bebiam. Entre as que bebiam, $30(15 \%)$ gostavam de beber de um a dois goles. Quando questionados sobre a possibilidade de encontrar ajuda para os problemas relacionados ao consumo de substâncias psicoativas, $99 \quad(49,5 \%)$ dos estudantes achavam que era possível conseguir ajuda na universidade $\mathrm{e}^{(16)}$.

Assim como demonstrado em outros estudos, o álcool é a substância mais consumida pelos estudantes, seguido do tabaco e da maconha. Dentre os motivos que levaram esses jovens a consumir bebidas alcoólicas estão a diversão e a fuga de seus problemas. $O$ beber socializador esteve presente entre os jovens. Quando questionados sobre a possibilidade de serem punidos se fossem pegos usando ou possuindo álcool e/ou outras drogas na universidade, menos da metade respondeu ser possível tal punição. Tal fato pode estar relacionado à inexistência de políticas relacionadas ao consumo de drogas no campus $^{(16)}$.

Pesquisa descritiva-correlacional desenvolvida com 80 estudantes teve por finalidade identificar se os universitários da área da saúde percebiam benefícios no uso do tabaco e outras drogas, além das dificuldades para deixarem de ser usuários. Do total da amostra, dois terços não fumavam; $16,3 \%$ fumaram por volta de 4 a 6 anos; e, 2,3\% fumavam há mais de 10 anos. Grande parte dos estudantes declarou não ter familiares que fumaram. A maioria considerou o uso do tabaco um problema, sendo importante alertar a comunidade sobre esse aspecto. Um terço dos estudantes respondeu que a conduta de não fumar foi uma opção pessoal, enquanto que o comportamento de fumar foi, por muitas vezes, influenciado pelos amigos ${ }^{(17)}$.

Em relação ao uso do álcool, mais de um terço da amostra respondeu que se embriagava uma vez ao mês. Aproximadamente dois terços 
dos participantes relataram que seus familiares não bebiam, e menos de um terço mencionaram que mais da metade são alcoólatras. Os benefícios proporcionados pelas drogas percebidos pela maioria dos estudantes foram o relaxamento, o prazer e a aceitação social. Dentre as barreiras para o abandono estavam o hábito e seus efeitos. Uma pequena parcela de estudantes não considerou o uso dessas substâncias como um problema de saúde e informaram o uso de outras substâncias na medida em que progrediram os anos de estudos. Para os autores dessa pesquisa, a inserção de temática de prevenção e reabilitação referentes ao uso de drogas nas disciplinas ministradas, durante 0 curso de graduação, resultará em impactos positivos no comportamento dos estudantes, motivando os futuros profissionais da saúde a manter condutas preventivas, bem como, incentivar sua autorresponsabilidade ${ }^{(17)}$.

Estudo do tipo epidemiológico de corte transversal teve como objetivo avaliar o consumo de SPA e seus determinantes pelos discentes do curso de enfermagem da Universidade de Passo Fundo, propondo a redução do uso. Participaram do estudo 266 estudantes matriculados na primeira semana do mês de março de 2007. Para coleta de dados utilizou-se um questionário sobre o uso de SPA do Centro Brasileiro de Informações sobre Drogas Psicotrópicas (CEBRID). Os resultados revelaram que a maioria dos participantes $(82 \%)$ era do sexo feminino, com renda familiar de dez salários mínimos e religião católica $(77,1 \%)$. O álcool foi a droga de maior experimentação seguida pelo tabaco, estimulantes e benzodiazepínicos. Dos 249 consumidores de bebidas alcoólicas, 69\% relataram embriaguez. Dentre os locais onde experimentaram bebidas alcoólicas, pela primeira vez, estavam os bares, danceterias e boates, casa dos amigos e familiares. Sendo estes últimos citados como os principais responsáveis por introduzir o álcool na vida dos acadêmicos. Uma evidência importante do estudo foi o fato de os estudantes considerarem, dentro do ambiente familiar, a figura do pai como o maior consumidor de bebidas alcoólicas. Os autores destacaram que os adolescentes e adultos jovens compõem uma população com maior probabilidade de consumir álcool, e a legitimação cultural conferida por várias sociedades a essa substância contribuiu para o consumo ${ }^{(18)}$.

Chama a atenção o consumo pesado realizado pelas acadêmicas, da amostra estudada e há uma preocupação com as usuárias de benzodiazepínicos que consumiam álcool, pois, essa associação pode levar ao estado de coma. O maior consumo desses medicamentos por mulheres pode ter ocorrido, em decorrência das dificuldades que apresentavam no enfrentamento de problemas originados no ambiente sociofamiliar, o que aumentaria a predisposição ao consumo dessas substâncias ${ }^{(18)}$.

Realizou-se um estudo de abordagem quantitativa, descritivo transversal cujo objetivo foi investigar o uso de substâncias psicoativas, lícitas e ilícitas, entre estudantes do curso de enfermagem noturno da PUC Minas, no intuito de discutir a diferença entre gênero no consumo, fornecendo assim, subsídio para futuros programas de prevenção. $O$ estudo foi composto por 393 estudantes e para a coleta de dados utilizou-se o questionário adaptado do instrumento proposto pela Organização Mundial de Saúde $(\mathrm{OMS})^{(19)}$.

Os autores verificaram que a prevalência do sexo feminino foi de 352 (89,5\%), assim como em outros estudos, cuja população alvo provém de cursos da área da saúde. O álcool $(89,3 \%)$ e o tabaco $(31,3 \%)$ foram as substâncias lícitas com predomínio do "uso na vida" e, no que se refere ao uso de substâncias psicoativas ilícitas, houve predomínio dos ansiolíticos (19,0\%), seguidos dos inalantes (15,5\%), anorexígenos (13,9\%) e maconha $(12,7 \%)$. Observaram que entre estudantes do sexo feminino há um maior consumo de ansiolíticos e anorexígenos em relação aos estudantes do sexo masculino ${ }^{(19)}$.

Um interessante fato identificado neste estudo foi o de que a experiência do uso do álcool apresentou-se maior entre os participantes do sexo feminino, como também a precocidade de início do consumo. Geralmente, a primeira experiência com álcool pelos estudantes de enfermagem ocorreu na adolescência, fato evidenciado em outras pesquisas, reforçando a ideia de que o consumo ocorre anteriormente ao ingresso na universidade ${ }^{(20)}$. Os dados identificados no presente estudo contrapõem-se aos evidenciados em outro estudo, no qual, apesar da prevalência de estudantes do sexo feminino no curso de Enfermagem, o maior consumo de bebidas alcoólicas esteve presente entre estudantes do sexo masculino ${ }^{(14)}$.

O fato de o consumo de ansiolíticos e anorexígenos ser mais expressivo no sexo feminino foi também evidenciado por uma outra pesquisa, na qual constatou-se o consumo 
pesado de benzodiazepínicos entre universitárias e o perigo de consumo dos mesmos associados ao álcool, pode levá-las ao estado de coma ${ }^{(18)}$.

Os fatores estressores na universidade, tais como, aumento da responsabilidade, ansiedade, tarefas acadêmicas, dificuldades financeiras e sociais, tendem a fazer com que os estudantes busquem no álcool e em outras drogas uma forma de aliviar as situações estressoras ${ }^{(19)}$.

Chama a atenção um estudo qualitativo que buscou compreender como se dá o processo saúde-doença dos estudantes que moravam no Conjunto Residencial da Universidade de São Paulo (CRUSP), quanto ao uso problemático de álcool e outras drogas, a fim de subsidiar direcionamento para ações preventivas e apoio voltado para essa população específica, considerando a diferença de gênero ${ }^{(20)}$.

A coleta foi realizada por meio de entrevista semiestruturada, que apresentava questões fechadas e abertas acerca da história do processo saúde-doença, durante a vida anterior e após o ingresso no CRUSP. Participaram do estudo oito estudantes homens e mulheres, moradores ou hóspedes regulares dos sete blocos de moradia que, na época da coleta de dados, haviam estado ou estavam envolvidos com o uso problemático de álcool e outras drogas ${ }^{(20)}$.

A análise dos dados foi embasada na Teoria da Determinação Social incorporando a categoria gênero. Da análise dos dados emergiram oito categorias empíricas: A droga na vida; Crusp como espaço favorecedor para uso de álcool e outras drogas; Uso de álcool e outras drogas: hereditariedade ou influência familiar; Uso de álcool e outras drogas como instrumento de fuga da realidade; Uso do álcool e outras drogas e discriminação; Uso problemático de álcool e outras drogas por homens e mulheres; Uso problemático de álcool e outras drogas e saúde mental; e Uso de álcool e outras drogas e violência ${ }^{(21)}$. As categorias empíricas trouxeram questões importantes como as diferenças relacionadas ao gênero. Na categoria "Uso de álcool e outras drogas e discriminação", por exemplo, sete sujeitos relataram ter sofrido algum tipo de discriminação. Alguns deles referiram-se a autodiscriminação, ou seja, reprovam a si mesmo cada vez que usam drogas de maneira abusiva. É importante ressaltar que as duas mulheres entrevistadas relataram ter uma grande preocupação com sua imagem frente à sociedade pelo uso problemático de álcool e outras drogas. Essa preocupação emergiu do fato de haver um preconceito social em relação às mulheres usuárias de drogas ${ }^{(20)}$.

Outra categoria importante foi "Uso problemático de álcool e outras drogas por homens e mulheres: diferença de sexo e de gênero". Nessa categoria evidenciou-se uma tendência três vezes maior dos homens se tornarem dependentes do álcool quando comparados às mulheres. Estas, por sua vez, apresentaram dependência associada ao uso de benzodiazepínicos e barbitúricos, fato que foi evidenciado em outro estudo ${ }^{(18)}$. O risco de as mulheres sofrerem abuso sexual quando intoxicadas foi manifestado pela maioria-dos participantes.

Segundo as autoras, há uma relação de subalternidade das mulheres em relação aos homens. Aquelas tendem a omitir a dependência química, em razão do preconceito, dificultando o diagnóstico e o tratamento. Outro dado marcante foi a culpabilidade das mulheres por serem usuárias de drogas, tanto por si mesma como pelos demais ${ }^{(20)}$.

As demais categorias revelaram situações já contextualizadas em outros estudos, tais como: início de consumo de álcool e ou outras drogas, em sua maioria, na adolescência; a liberdade de acesso às drogas que a moradia proporciona; questões relacionadas à influência familiar e à violência. Para as autoras, a pesquisa evidenciou que a moradia universitária não apareceu como elemento responsável por desencadear o consumo de álcool e outras drogas, mas como mais um elemento favorecedor ${ }^{(20)}$.

Como evidenciado nos estudos acima, o uso de substâncias psicoativas entre os acadêmicos é caracterizado como um sério problema de saúde pública, que requer a implantação de políticas coerentes advindas do Estado e das instituições de ensino para o melhor enfrentamento, por parte dos discentes, no que diz respeito ao uso e abuso das SPA, às ações de prevenção e de tratamento ${ }^{(21)}$.

Verificou-se que o álcool é a droga de maior consumo entre os estudantes. No entanto, o consumo de benzodiazepínicos e anfetamínicos, principalmente por mulheres, foi um achado de alguns estudos ${ }^{(6,18-19)}$. Outro achado importante se referiu ao início do consumo, uma vez que, a maioria dos estudos relatou ser anteriormente ao ingresso na universidade.

Destaca-se, ainda, no presente estudo, as diferenças de gênero e a intensidade com que os padrões sociais podem afetá-los. Diferentemente 
dos demais estudos que buscaram, por meio de instrumentos de coleta, verificar os hábitos, reações, atitudes e opiniões do público alvo ${ }^{(20)}$.

No geral, os estudos, apontaram um aumento do consumo de álcool e outras drogas entre mulheres. Tal resultado corrobora com dados do Relatório da Organização das Nações Unidas (ONU) que destaca que o aumento da renda e o estresse serão fatores essenciais para que as mulheres cheguem ao patamar de consumo semelhante aos homens até $2020^{(3)}$.

Embora exista uma preocupação entre os pesquisadores em relação ao uso de álcool e/ou outras drogas entre universitários, ficou evidente que o início do consumo dessas substâncias entre universitários não se encontra, necessariamente, vinculado ao ingresso na universidade. Tal afirmação é retratada nos estudos aqui expostos, nos quais os graduandos referiram a primeira experiência com bebidas alcoólicas na faixa etária de 13 a 18 anos.

Ainda que o ambiente universitário não propicie o início do consumo, há de se considerar que 0 uso de SPA entre acadêmicos pode ser intensificado, ou até mesmo estimulado, em decorrência do processo de mudança vivenciado durante essa fase da vida.

Alguns fatores se articulam para que os estudantes universitários sejam considerados vulneráveis ao uso de álcool e/ou drogas dentre os quais encontram-se, estressores relacionados as exigências acadêmicas, a necessidade de estabelecimento de interações sociais e integração com os demais universitários nos mais diferentes contextos, além da disponibilidade de substâncias na maioria dos eventos recreativos e lazer dessa população.

Outros fatores como o distanciamento do núcleo familiar, sobrecarga de atividades e permissividade de condutas também se encontram atrelados à adesão ou intensificação do consumo dessas substâncias. Assim sendo, entende-se que o consumo de substâncias psicoativas, nesse grupo, se dá, muitas vezes, como estratégia de enfrentamento desses estressores.

Destacam-se ainda, os comportamentos de risco advindos do consumo dessas substâncias, aos quais os jovens estão sujeitos, como relações sexuais desprotegidas que podem resultar em gravidez indesejada e infecções sexualmente transmissíveis (IST's), agressão e acidentes automobilísticos. O consumo desregrado de substâncias psicoativas corrobora para um quadro de dependência que, conforme a gravidade, pode resultar em depressão, tentativa de suicídio e morte.

O fenômeno de consumo de substâncias psicoativas é algo preocupante que exige acompanhamento dos órgãos responsáveis

Apreende-se, a partir dos estudos, que o consumo de álcool e de SPA é uma realidade entre os universitários, principalmente entre os homens e as questões sociais, culturais e econômicas constituem os fatores para o seu consumo.

\section{CONCLUSÃO}

A reflexão teórica realizada possibilitou apresentar o panorama de consumo de álcool e outras drogas entre estudantes universitários. Embora muitos estudos evidenciem que o álcool é a droga mais consumida, e que o início do uso dessas substâncias não ocorre, necessariamente, ao ingressar na universidade, nota-se a partir dos estudos, que o consumo pode ser intensificado ou, até mesmo, estimulado, em razão de todo o processo de mudança que perpassa a vida desses jovens.

O consumo cada vez mais desregrado dessas substâncias tem, como consequência, o surgimento de comportamentos de risco que podem marcar a vida desses estudantes, além de corroborar para um quadro efetivo de dependência química.

Ainda que para o senso comum o ingresso no ensino superior esteja relacionado ao crescimento profissional, existe um outro lado que o Estado brasileiro e as instituições de ensino não podem negligenciar. Compete ao Estado brasileiro a elaboração e o incentivo para o cumprimento de políticas públicas que minimizem o consumo de SPA entre jovens, especialmente, universitários.

À universidade compete a educação para a autonomia não apenas profissional, mas também a implementação de atividades e oportunidades, para além da sala de aula, que possam motivar a sua participação e promover ações que contribuam para a satisfação dos universitários. Compete ainda, o respeito pelas escolhas, o acolhimento, a escuta qualificada e o encaminhamento aos profissionais, quando necessário.

Uma das limitações desse estudo é a escassez de publicações de abordagem qualitativa que visam compreender os motivos pelos quais os universitários têm aumentado o 
consumo de álcool e/ou drogas, além de enfatizar as questões de gênero em relação a esse consumo.

As reflexões que emergem deste estudo despertam para a necessidade de realização de novas pesquisas que tenham como foco a criação e desenvolvimento de programas de intervenção efetivos que busquem a promoção da saúde entre estudantes universitários, considerando a vulnerabilidade desse grupo ao consumo de álcool e/ou drogas.

Ademais como contribuição, este estudo traz a necessidade de criar políticas efetivas no ambiente acadêmico para o enfrentamento das questões referentes ao uso dessas substâncias, com vistas às ações de prevenção e de tratamento, que contemplem as reais necessidades dos jovens universitários, sem nenhum tipo de repressão e de discriminação.

\section{REFERÊNCIAS}

1. Brasil. Ministério da Saúde. A Política do Ministério da Saúde para atenção integral a usuários de álcool e outras drogas. 2. ed. rev. e ampl. Brasília: Ministério da Saúde; 2004.

2. Henriquéz PC, Carvalho AMP. Percepção dos benefícios do consumo de drogas e das barreiras para seu abandono entre estudantes da área da saúde. Rev. Latino-Am Enfermagem 2008;16 (nesp):621-6. DOI: $\quad$ 10.1590/S010411692008000700019

3. United Nations Office on Drugs and Crime. World Drug Report 2014. New York: United Nations; 2014.

4. Silva EF, Pavani RAB, Moraes MS, Chiaravalloti Neto F. Prevalência do uso de drogas entre escolares do ensino médio do Município de São José do Rio Preto, São Paulo, Brasil. Cad. Saúde Pública 2006;22(6):1151-8. DOI: 10.1590/S0102311X2006000600004

5. García NAA, Luis MAV, Castillo MMA, Castillo BAA, Rodríguez NNO. Eventos estresantes y su relación con el consumo de alcohol en estudiantes universitarios. Investig Enferm Imagen Desarr. 2013 [citado em 15 jan 2017];14(2):97-112. Disponível em: http://www.redalyc.org/articulo.oa?id=14522675 $\underline{8007}$

6. Nóbrega MPSS, Simich L, Strike C, Brands B, Giesbrecht N, Khenti A. Policonsumo simultâneo de drogas entre estudantes de graduação da área de ciências da saúde de uma universidade: implicações de gênero, sociais e legais, Santo André-Brasil. Texto Contexto - Enferm. 2012;21
(1):25-33. DOI: $\quad \underline{10.1590 / 50104-}$ $\underline{07072012000500003}$

7. Wagner GA, Andrade AG. Uso de álcool, tabaco e outras drogas entre estudantes universitários brasileiros. Rev Psiquiatr Clín. 2008;35(supl 1):48-54. DOI: 10.1590/S010160832008000700011

8. Eckschmidt, Andrade AG, Oliveira LG. Comparação do uso de drogas entre universitários brasileiros, norte-americanos e jovens da população geral brasileira. J Bras Psiquiatr. 2013;62(3):199-207. DOI: 10.1590/S0047-20852013000300004

9. Brasil. Presidência da República. Secretaria Nacional de Políticas sobre Drogas. I Levantamento nacional sobre o uso de álcool, tabaco e outras drogas entre universitários, realizado nas 27 capitais brasileiras. Brasília: SENAD; 2010.

10.Fachini, A, Furtado, EF. Uso de álcool e expectativas do beber entre universitários: uma análise das diferenças entre os sexos. Psic: teor e pesq. 2013;29(4):196-223. DOI: 10.1590/S0102$\underline{37722013000400008}$

11.Ortega-Pérez CA, Costa-Júnior ML, Vasters GP. Perfil epidemiológico de la drogadicción en estudiantes universitarios. Rev Latino-Am Enfermagem 2011;19(nesp):665-72. DOI: http://www.scielo.br/scielo.php?script=sci arttex t\&pid=S0104-11692011000700002

12.Pereira MO, Cardoso CS, Coata LMCG, Sampaio VM, Oliveira MAF. O consumo de álcool e outras drogas entre estudantes universitários: interferências na vida acadêmica. SMAD, Rev. Eletrônica Saúde Mental Álcool Drog., 2013, set./dez; 9(3): 105-10. DOI: 10.11606/issn.18066976.v9i3p105-110

13.Freitas RLM, Nascimento DS, Freitas RM, Saldanha GB, Rocha RMM, Santos PS. Perfil da utilização de drogas lícitas e ilícitas por universitários de uma instituição privada, SMAD, Rev. Eletrônica Saúde Mental Álcool Drog. 2012;8(3):118-26. DOI: 10.11606/issn.18066976.v8i3p118-126

14.Pillon SC, Santos MA, Gonçalves MAS, Araújo $\mathrm{KM}$, Funai A. Fatores de risco, níveis de espiritualidade e uso de álcool em estudantes de dois cursos de enfermagem. SMAD, Rev. Eletrônica Saúde Mental Álcool Drog. 2010; 6(nesp):493-513. DOI: 10.11606/issn.18066976.v6ispep493-513

15.Baumgarten LZ, Gomes VLO, Fonseca AD. Consumo alcoólico entre universitários (as) da área da saúde da Universidade Federal do Rio 
Grande/RS: subsídios para enfermagem. Esc Anna Nery 2012;16(3):530-5. DOI: 10.1590/S141481452012000300015

16.Pillon SC, O'Brien B, Chavez KAP. A relação entre o uso de drogas e comportamentos de risco entre universitários brasileiros. Rev Latino-Am Enfermagem 2005;13(nesp 2):1169-76. DOI: 10.1590/S0104-11692005000800011

17. Henriquéz, PC, De Carvalho, AMP. Percepção dos benefícios do consumo de drogas e das barreiras para seu abandono entre estudantes da área da saúde. Rev. Latino-Am Enfermagem 2008;16(nesp):621-6. DOI: $10.1590 / S 0104-$ 11692008000700019

18.Picolotto E, Libardoni LFC, Migotti AMB, Geib LTC. Prevalência e fatores associados com o consumo de substâncias psicoativas por acadêmicos de enfermagem da Universidade de Passo Fundo. Ciênc Saúde Coletiva 2010;15(3):645-54. DOI: 10.1590/S1413$\underline{81232010000300006}$

19.Botti NCL, Lima AFD, Simões WMB. Uso de substâncias psicoativas entre acadêmicos de enfermagem da Universidade Católica de Minas Gerais. SMAD, Rev. Eletrônica Saúde Mental Álcool Drog. 2010;6(1):1-16. DOI: 10.11606/issn.1806-6976.v6i1p1-20

20.Zalaf MRR, Fonseca RMGS. Uso problemático de álcool e outras drogas em moradia estudantil: conhecer para enfrentar. Rev Esc Enferm USP 2009;43(1):132-8. DOI: $10.1590 / 50080-$ $\underline{62342009000100017}$

21.Gonçalves JS, Dázio EMR, Fava SMC. Significado da droga para as universitárias. Rev Enferm UFPE 2017;11(supl 10):4136-42. DOI: $\underline{\text { 10712-95194-3-SM.1110sup201717 }}$

Nota: O artigo constitui-se parte da Dissertação de Mestrado intitulada "Significado do uso de álcool e/ou drogas entre universitárias".

Recebido em: 04/11/2017

Aprovado em: 08/07/2019

Endereço de correspondência:

Jamila Souza Gonçalves

Rua Mário Ribola, 409 - Penha II

CEP: 37903-358 - Passos/MG - Brasil

E- mail: jamila.goncalves@ifsuldeminas.edu.br 